\title{
Exponential Stability Criteria for Nonautonomous Difference Systems
}

\author{
Rigoberto Medina \\ Departamento de Ciencias Exactas, Universidad de Los Lagos, Casilla Postal 933, 5290000 Osorno, Chile \\ Correspondence should be addressed to Rigoberto Medina; rmedina@ulagos.cl
}

Received 28 January 2014; Revised 25 March 2014; Accepted 9 April 2014; Published 29 April 2014

Academic Editor: Henryk Hudzik

Copyright ( 2014 Rigoberto Medina. This is an open access article distributed under the Creative Commons Attribution License, which permits unrestricted use, distribution, and reproduction in any medium, provided the original work is properly cited.

\begin{abstract}
The aim of this paper is to characterize the exponential stability of linear systems of difference equations with slowly varying coefficients. Our approach is based on the generalization of the freezing method for difference equations combined with new estimates for the norm of bounded linear operators. The main novelty of this work is that we use estimates for the absolute values of entries of a matrix-valued function, instead of bounds on its eigenvalues. By this method, new explicit stability criteria for linear nonautonomous systems are derived.
\end{abstract}

\section{Introduction}

In the theory of difference equations it is well known that the placement of eigenvalues in the complex plane of a timeinvariant linear system is a necessary and sufficient condition to ensure the stability or exponential stability. However, in time-varying systems, the stability and exponential stability are not characterized by the spectrum of transition matrices (see, e.g., [1, 2]). Desoer [1] illustrated the same instability characteristic of a class of discrete-time-varying systems, but remedied the situation considering bounded and sufficiently slowly varying coefficients. More explicitly, Desoer considered the system in $R^{n}$ (the Euclidean $n$-dimensional space):

$$
x(t+1)=A(t) x(t), \quad t=0,1, \ldots,
$$

where $x(t) \in R^{n}$ and $A(t) \in R^{n \times n}$ for all $t\left(R^{n \times n}\right.$ denotes the class of $n \times n$-matrices with real elements), and his assumptions are as follows:

(a) there is a finite $a_{M}$ such that

$$
\sup _{t \geq 0}\|A(t)\|=a_{M}<\infty,
$$

(b) for some $\varepsilon>0$,

$$
\max _{j \geq 0}\left|\lambda_{j}(A(t))\right| \leq 1-2 \varepsilon<1
$$

(c) $\sup _{t \geq 0}\|A(t+1)-A(t)\|$ is sufficiently small.
Under this set of conditions it is proven that system (1) is exponentially stable. Actually, Desoer uses (a) and (b) to generate a bound of the form

$$
\left\|A^{m}(t)\right\| \leq M \rho^{m} ; \quad \forall t, \forall m \geq 1
$$

where $\rho=1-\varepsilon$ and $M$ depends on $\varepsilon, n$ and $a_{M}$ but is independent of $t$, and then uses a Lyapunov argument to show that system (1) is exponentially stable if $\sup _{t \geq 0}\|A(t+1)-A(t)\|$ is small enough.

Without the restriction on the rate of variation on $A(t)$, the system (1) may have exponentially increasing solutions. Thus, there must be an additional condition on $A(t)$ in order to get stability.

It is well known that the Lyapunov function method serves as a main technique to reduce a given complicated system into a relatively simpler system, and it provides useful applications to control theory, but finding Lyapunov functions is still a difficult task (see, e.g., [1, 3-6]). By contrast, many methods different from Lyapunov functions have been successfully applied to the stability analysis of discrete-time systems (see, e.g., [1, 2, 5, 7-10]).

Recently, Gil and Medina [11, 12] and Medina [13-15] begun the study of stability and stabilizability theory for discrete-time systems by means of new estimates for the powers of matrix-valued and operator-valued functions. 
In the last decades many investigations have dealt with exponential stability conditions for discrete-time-varying systems [6-8, 16-19] or continuous time-varying systems [2, 7-9].

Our aim is to relax (b) by using estimates for the absolute values of entries of a matrix-valued function, instead of bound on its eigenvalues. Our proof technique is based on the generalization of the "freezing" method for difference equations (see, e.g., Gil and Medina [11]) combined with new estimates for the norm of powers of variable matrices.

If $A(t)$ is periodic, then the discrete Floquet theory [18] provides necessary and sufficient conditions for stability. However, the Floquet approach requires calculation of the Monodromy matrix, which is generally a difficult task. Thus, our results are of relevance even in the periodic case.

To the best of our knowledge, this work is the first using the above mentioned approach to develop a theory concerning the exponential stability of linear time-varying discrete systems. Besides our results being explicit, they are easy to verify. Moreover, the solutions' estimates give us the possibility to investigate linear and nonlinear perturbations of system (1). Indeed, this approach has little overlapping with the existing literature, mainly because our results do not require solving the characteristic polynomial associated with the variable matrices $A(t)$, which in turn is a difficult task for higher dimensional systems.

The structure of this paper is as follows. In Section 2, we introduce some notations and the fundamental results concerning estimates for the absolute values of entries of matrix-valued functions of finite matrices. In Section 3, exponential stability results and its consequences are established for nonautonomous discrete-time systems. In Section 4, as an application of the previous results, we characterize the exponential stability of nonlinear perturbations of system (1). Finally, Section 5 is devoted to the discussion of our results: we highlight the main conclusions and state some directions for future research.

\section{Preliminaries and Problem Statement}

Let $C^{n}$ be a complex Euclidean space with the scalar product $(\cdot, \cdot)$, as well as the unit matrix $I$. Let $\sigma(A)$ be the spectrum of a linear operator (a matrix) $A$ and

$$
R_{z}(A)=(A-z I)^{-1}, \quad(z \notin \sigma(A)),
$$

the resolvent of $A$. For a scalar valued function $f(\lambda)$, holomorphic on the spectrum of $A$, the matrix-valued function $f(A)$ is defined by

$$
f(A)=-\frac{1}{2 \pi i} \int_{\Gamma} f(\lambda) R_{\lambda}(A) d \lambda,
$$

where $\Gamma$ is a closed contour surrounding $\sigma(A)$.

Let $\left\{e_{k}\right\}_{k=1}^{n}$ be a fixed orthonormal basis in $C^{n}$ and $a_{j k}(j, k=1,2, \ldots, n)$ the entries of a matrix $A$ in this basis.

We put $|A|=\left(\left|a_{j k}\right|\right)_{j, k=1}^{n}$; that is, $|A|$ is a matrix whose entries are absolute values of $A$ in $\left\{e_{k}\right\}_{k=1}^{n}$. If $h \in C^{n}$, then $|h|=\left(\left|h_{11}\right|, \ldots,\left|h_{1 n}\right|\right) \in C^{n}$.
Clearly, $A=D+V$, where $D=\operatorname{diag}\left\{a_{11}, \ldots, a_{n n}\right\}$ is the diagonal of $A$ and $V=A-D$ is the off-diagonal part of $A$; that is, the entries $v_{j k}$ of $V$ are $v_{j k}=a_{j k}($ if $j \neq k)$ and $v_{j j}=0$.

Denote by $r_{S}(T)$ the spectral radius of an operator $T$. Clearly,

$$
r_{S}(D)=\max _{1 \leq j \leq n}\left|a_{j j}\right|
$$

Thanks to the well-known inequality for the spectral radius, we have

$$
r_{S}(A) \leq \tilde{r}_{A}
$$

where

$$
\tilde{r}_{A}=\max _{1 \leq j \leq n}\left(\sum_{k=1}^{n}\left|a_{j k}\right|\right) .
$$

Denote by $c_{0}(D)$ the closed convex hull of the diagonal entries $a_{11}, \ldots, a_{n n}$.

Theorem 1 (see $[10,20])$. Let $V$ be the off-diagonal part of an $n \times n$-matrix A. Let $f(\lambda)$ be holomorphic on a neighborhood of the circle

$$
\Omega(A)=\left\{z \in C:|z| \leq \widetilde{r}_{A}\right\} .
$$

Then, with the notation

$$
\gamma_{k}(A)=\sup _{z \in c_{0}(D)} \frac{\left|f^{(k)}(z)\right|}{k !}, \quad k=0,1, \ldots,
$$

the matrix inequality

$$
|f(A)| \leq \sum_{k=0}^{\infty} \gamma_{k}|V|^{k}
$$

holds, provided the series in (12) converges.

Note that, according to (9), we have the inequality

$$
r_{S}(|V|) \leq \max _{1 \leq j \leq n}\left(\sum_{k=1, j \neq k}^{n}\left|a_{j k}\right|\right) .
$$

Definition 2. A norm $\|\cdot\|$ in $C^{n}$ is said to be monotone if for all $h, g \in C^{n}$ we have $\|h\| \leq\||h|\|$; and $\|h\| \leq\|g\|$, provided $|h| \leq|g|$.

Corollary 3 (see $[10,20]$ ). Let $f(\lambda)$ be holomorphic on a neighborhood of $\Omega(A)$. Let $\|\cdot\|$ be an arbitrary monotone norm in $C^{n}$. Then

$$
\|f(A)\| \leq \sum_{k=0}^{\infty} \gamma_{k}\left\||V|^{k}\right\|
$$

provided the series in (14) converges.

Important contributions to the theory of matrix-valued functions can be found in Verde-Star [21]. 
Definition 4. The zero solution of (1) is said to be exponentially stable if there exist $\delta>0, M>0$, and $0<\eta<1$ such that

$$
\left\|x\left(t, t_{0}, x_{0}\right)\right\| \leq M \eta^{t-t_{0}}\left\|x_{0}\right\|, \quad \text { whenever }\left\|x_{0}\right\|<\delta,
$$

with $x\left(t_{0}\right)=x_{0}$

Let $\Delta=\left\{(t, s) \in Z^{+} \times Z^{+}: t \geq s\right\}$, with $Z^{+}$being the set of nonnegative integers, and let $\Phi=\{\Phi(t, s)\}_{(t, s) \in \Delta}$ be the evolution operator associated with the system (1); that is,

$$
\Phi(t, s)= \begin{cases}A(t-1) \cdots A(s), & t>s \\ I, & \text { if } t=s .\end{cases}
$$

Definition 5. The zero solution of system (1) is uniformly exponentially stable if there exist positive constants $M$ and $0<\eta<1$ such that

$$
\|\Phi(t, s)\| \leq M \eta^{t-s}, \quad \text { for } t_{0} \leq s \leq t<\infty .
$$

Now, we are in a position to establish the main results of this work.

\section{Main Results}

Consider in $R^{n}$ the system

$$
x(t+1)=A(t) x(t), \quad(t=0,1, \ldots)
$$

with the initial condition

$$
x(s)=x_{0},
$$

with a given $x_{0} \in R^{n}$ and a fixed $s \geq 0$.

Assume that there exists a nonnegative sequence $q(t)$ such that

$$
\|A(t)-A(k)\| \leq q(t-k), \quad \text { for } t \geq s \geq 0,
$$

where $q(0)=0$.

Firstly, we recall a boundedness result for (18)-(19) which is proven in [22, Lemma 1.1]; namely, we recall the following lemma.

Theorem 6 (see [11]). Under (20), let

$$
\xi_{0}=\sum_{t=1}^{\infty} q(t) \sup _{l=0,1, \ldots}\left\|A^{t}(l)\right\|<1 .
$$

Then, every solution $x(t)$ of (18)-(19) satisfies the inequality

$$
\sup _{t=1,2, \ldots}\|x(t)\| \leq \frac{\beta_{0}\left\|x_{0}\right\|}{1-\xi_{0}},
$$

where

$$
\beta_{0}=\sup _{t \geq 0}\left(\sup _{l \geq 0}\left\|A^{t}(l)\right\|\right)
$$

Theorem 7 (see [12]). Assume that there exists $\tilde{q}>0$ such that

$$
\begin{gathered}
\|A(t)-A(k)\| \leq \widetilde{q}|t-k|, \quad(t, k=0,1, \ldots), \\
\widetilde{\xi}_{0}=\sum_{t=1}^{\infty} t \sup _{l=0,1, \ldots}\left\|A^{t}(l)\right\|<\frac{1}{\widetilde{q}} .
\end{gathered}
$$

Then (18)-(19) is uniformly stable. Moreover, any solution $x(t)$ of (18) satisfies the inequality

$$
\|x(t)\| \leq \frac{\beta_{0}\|x(s)\|}{1-\tilde{q} \tilde{\xi}_{0}}, \quad t=s+1, s+2, \ldots
$$

Corollary 8 (see [12]). Let condition (24) hold. In addition, for a constant $v \in(0,1)$, let

$$
\theta(v)=\sum_{t=1}^{\infty} t v^{-t-1} \sup _{l=0,1, \ldots}\left\|A^{t}(l)\right\|<\frac{1}{\widetilde{q}}
$$

Then (18)-(19) is exponentially stable. Moreover, any solution $x(t)$ of (18) satisfies the inequality

$$
\|x(t)\| \leq \frac{v^{t-s} \widetilde{m}(v)\|x(s)\|}{1-\tilde{q} \theta(v)}, \quad t=s+1, s+2, \ldots,
$$

where $\widetilde{m}(v)=\sup _{t \geq 0}\left(\sup _{l \geq 0}\left(\left\|A^{t}(l)\right\| / v^{t}\right)\right)$.

In fact, due to (25), $\vec{m}(v)<\infty$. Putting in (18),

$$
x(t)=v^{t} z(t),
$$

one has

$$
z(t+1)=\frac{1}{v} A(t) z(t) .
$$

Hence, by conditions (24) and (26), one has

$$
\|z(t)\| \leq \frac{\widetilde{m}(v)\|z(s)\|}{1-q_{0} \theta(v)}, \quad t=s+1, s+2, \ldots,
$$

because of the fact that if $\widetilde{A}(t)=A(t) / v$, then $\|\widetilde{A}(t)-\widetilde{A}(t+1)\| \leq$ $q_{0} / v$.

Remark 9. Condition (20), combined with the freezing technique, allows us to reduce the stability analysis of a time-varying system to the analysis of the related timeinvariant system, thus exploiting the tools developed for linear autonomous systems. However, this condition is conservative compared with the approach proposed by Jetto and Orsini $[2,8]$, which does not require slowly varying conditions on the coefficients.

Our main results are made possible by the following bound.

Lemma 10. Let $V$ be the off-diagonal part of an $n \times n$-matrix $A$. Then, for an arbitrary monotone norm $\|\cdot\|$ in $C^{n}$,

$$
\left\|A^{m}\right\| \leq\left(r_{S}(D)+\||V|\|\right)^{m}, \quad \text { for } m=1,2, \ldots .
$$


Proof. In particular, if in Theorem 1 we consider the matrixvalued function

$$
f(A)=A^{m}, \quad m=1,2, \ldots,
$$

where $A$ is an $n \times n$ constant matrix, hence,

$$
f^{(k)}(\lambda)= \begin{cases}\frac{m !}{(m-k) !} \lambda^{m-k}, & 0 \leq k \leq m \\ 0, & k>m .\end{cases}
$$

Thus, for every $\lambda \in c_{0}(D)$, relation (11) yields

$$
\gamma_{k}=\frac{m !}{(m-k) ! k !} r_{S}^{m-k}(D), \quad k=0,1, \ldots, m,
$$

where $A=D+V$. Thus, by (12),

$$
\left|A^{m}\right| \leq \sum_{k=0}^{m} \frac{m !}{(m-k) ! k !} r_{S}^{m-k}(D)|V|^{k}=\left(r_{S}(D) I+|V|\right)^{m},
$$

where $r_{S}(D)=\max _{1 \leq j \leq n}\left|a_{j j}\right|$. Hence, by (14), we have

$$
\left\|A^{m}\right\| \leq\left\|\sum_{k=0}^{m} \gamma_{k}|V|^{k}\right\| \leq \sum_{k=0}^{m} \gamma_{k}\left\||V|^{k}\right\| \leq\left(r_{S}(D)+\||V|\|\right)^{m} .
$$

As a consequence of Lemma 10, we obtain

$$
\left\|A^{k}(l)\right\| \leq\left(r_{S}(D(l))+\||V(l)|\|\right)^{k}, \quad \text { for fixed } l,
$$

where

$$
r_{S}(D(l))=\max _{1 \leq j \leq n}\left|a_{j j}(l)\right|, \quad l \text { fixed. }
$$

Assume that

$$
\rho_{0}=\sup _{l=0,1, \ldots}\left(r_{S}(D(l))+\||V(l)|\|\right) .
$$

Hence

$$
\xi_{0}=\sum_{t=0}^{\infty} q(t) \sup _{l=0,1, \ldots}\left\|A^{t}(l)\right\| \leq \sum_{t=0}^{\infty} q(t) \rho_{0}^{t} .
$$

Consequently,

$$
\sum_{t=0}^{\infty} q(t) \sup _{l=0,1, \ldots}\left\|A^{t}(l)\right\| \leq C\left(\rho_{0}\right),
$$

where $C\left(\rho_{0}\right)=\sum_{t=0}^{\infty} q(t) \rho_{0}^{t}$.

Theorem 11. Under (20), let

$$
C\left(\rho_{0}\right)=\sum_{t=0}^{\infty} q(t) \rho_{0}^{t}<1 .
$$

Then (18)-(19) is exponentially stable. Moreover, any solution $x(t)$ of (18) satisfies the inequality

$$
\|x(t)\| \leq \frac{\beta_{0}\|x(s)\|}{1-C\left(\rho_{0}\right)}, \quad(t \geq s \geq 0) .
$$

Proof. By (43), $\beta_{0}<\infty$. Hence, the uniform stability is a consequence of Theorem 1 . To establish the exponential stability of (18)-(19), let us define a new variable

$$
x(t)=\frac{y(t)}{(1+\varepsilon)^{t}}, \quad t=0,1, \ldots,
$$

with $\varepsilon>0$ small enough, where $x(t)$ is a solution of (18). Substituting (45) in (18), we have

$$
y_{\varepsilon}(t+1)=\widetilde{A}(t) y_{\varepsilon}(t), \quad t \geq 0,
$$

where $\widetilde{A}(t)=(1+\varepsilon) A(t)$.

By (22) and Theorem 6, we obtain the estimate (43). It provides the stability of (46). Besides, the boundedness of $y_{\varepsilon}(t)$, for $\varepsilon$ small enough, implies the exponential stability of $x(t)$.

Remark 12. In the particular case $\rho_{0}<1$, the system (1) is exponentially stable because in this situation we have

$$
\sup _{t \geq 0}\|A(t)\| \leq \rho_{0}<1,
$$

and no further conditions are needed.

\section{Application to Nonlinear Equations}

The previous estimates give us the possibility to investigate the stability of nonlinear perturbations of system (1).

Consider the equation

$$
x(t+1)=A(t) x(t)+F(t, x(t)), \quad t=0,1, \ldots,
$$

where $F$ maps $C^{n} \times Z^{+}$into $C^{n}$, with the property

$$
\|F(t, u)\| \leq \gamma\|u\|, \quad \forall t \in Z^{+}, u \in C^{n},
$$

where $Z^{+}$is the set of nonnegative integers. Take the initial condition

$$
x(0)=x_{0} \in C^{n} .
$$

Theorem 13. Under conditions (20) and (49), let

$$
S_{0}=\sum_{t=0}^{\infty}(q(t)+\gamma) \sup _{l=0,1, \ldots}\left\|A^{t}(l)\right\|<1 .
$$

Then the zero solution of (48)-(50) is exponentially stable. Moreover, a solution of (48), with $x(0)=x_{0}$, satisfies the inequality

$$
\sup _{t \geq 1}\|x(t)\| \leq \frac{\beta_{0}\left\|x_{0}\right\|}{1-S_{0}}
$$

where $\beta_{0}=\sup _{t \geq 0}\left(\sup _{l \geq 0}\left\|A^{t}(l)\right\|\right)$.

Proof. Given a fixed integer $s \geq 0$, we can write (48) in the form

$$
x(t+1)-A(s) x(t)=(A(t)-A(s)) x(t)+F(t, x(t)) .
$$


The variation of constants formula yields

$$
\begin{aligned}
x(m+1) & \\
= & A^{m+1}(s) x(0) \\
& +\sum_{j=0}^{m} A^{m-j}(s)[(A(j)-A(s)) x(j)+F(j, x(j))] .
\end{aligned}
$$

Taking $s=m$, we have

$$
\begin{aligned}
x(m+1) & \\
= & A^{m+1}(m) x(0) \\
& +\sum_{j=0}^{m} A^{m-j}(m)[(A(j)-A(m)) x(j)+F(j, x(j))] .
\end{aligned}
$$

Hence,

$$
\begin{aligned}
& \|x(m+1)\| \\
& \leq \beta_{0}\|x(0)\| \\
& \quad+\sum_{j=0}^{m}\left\|A^{m-j}(m)\right\|[q(m-j)\|x(j)\|+\gamma\|x(j)\|] \\
& \leq \beta_{0}\|x(0)\|+\max _{0 \leq t \leq m}\|x(t)\| \sum_{j=0}^{m}\left\|A^{m-j}(m)\right\|(q(j)+\gamma) .
\end{aligned}
$$

This inequality yields

$$
\|x(m+1)\| \leq \beta_{0}\|x(0)\|
$$

$$
+\max _{0 \leq t \leq m}\|x(t)\|\left(\sum_{j=0}^{\infty}(q(j)+\gamma) \sup _{l=0,1, \ldots}\left\|A^{j}(l)\right\|\right) .
$$

Thus,

$$
\max _{0 \leq t \leq m+1}\|x(t)\| \leq \beta_{0}\|x(0)\|+S_{0} \max _{0 \leq t \leq m+1}\|x(t)\| .
$$

From this relation, we obtain

$$
\max _{0 \leq t \leq m+1}\|x(t)\| \leq \frac{\beta_{0}\|x(0)\|}{1-S_{0}} .
$$

But the right-hand side of this inequality does not depend on $m$. Thus, it follows that

$$
\sup _{t=1,2, \ldots}\|x(t)\| \leq \frac{\beta_{0}\|x(0)\|}{1-S_{0}} .
$$

Bound (52) proves the Lyapunov stability with $\|x(0)\|$ small enough. To establish the exponential stability of the zero solution of system (48), we take the new variable

$$
x_{\varepsilon}(t)=x(t) e^{\varepsilon t}, \quad t \geq 0,
$$

with $\varepsilon>0$ small enough. Here $x(t)$ is the solution of (48)(50).
Substituting (61) in (48), we have

$$
x_{\varepsilon}(t+1)=\widetilde{A}(t) x_{\varepsilon}(t)+F_{1}\left(t, x_{\varepsilon}(t)\right),
$$

where

$$
\begin{aligned}
\widetilde{A}(t) & =e^{\varepsilon} A(t), \\
F_{1}(t, v) & =e^{(1+t) \varepsilon} F\left(t, e^{-\varepsilon t}\right), \quad v \in C^{n} .
\end{aligned}
$$

The growth condition (49) yields

$$
\left\|F_{1}(t, v)\right\| \leq \gamma e^{\varepsilon}\|v\|, \quad \forall v \in C^{n}, t=0,1, \ldots
$$

Applying the above reasoning to (62), according to (52), it follows that $x_{\varepsilon}(t)$ is a bounded function for $\varepsilon>0$ small enough. Consequently, relation (61) implies the exponential stability of the zero solution of (48).

The estimates established in Section 3 give us the possibility to improve condition (51) and estimate (52) of Theorem 13.

Theorem 14. Under conditions (20) and (49), let

$$
\widetilde{S}_{0}=\sum_{t=0}^{\infty}(q(t)+\gamma) \rho_{0}^{t}<1 .
$$

Then, the zero solution of (48) is exponentially stable. Moreover, a solution $x(t)$, with $x(0)=x_{0}$, satisfies the inequality

$$
\sup _{t=1,2, \ldots}\|x(t)\| \leq \frac{\beta_{0}\left\|x_{0}\right\|}{1-\widetilde{S}_{0}},
$$

where $\beta=\sup _{t \geq 0}\left(\sup _{l \geq 0}\left\|A^{t}(l)\right\|\right)$.

Proof. By (40) and (51), for any monotone norm, we have

$$
\sum_{t=0}^{\infty}(q(t)+\gamma) \sup _{l=0,1, \ldots}\left\|A^{t}(l)\right\| \leq \sum_{t=0}^{\infty}(q(t)+\gamma) \rho_{0}^{t} .
$$

Consequently, if $\sum_{t=0}^{\infty}(q(t)+\gamma) \rho_{0}^{t}<1$, then, by Theorem 13, the zero solution of (48) is exponentially stable.

Example 15. Consider the following nonautonomous difference system in $R^{2}$ :

$$
x(t+1)=A(t) x(t)+F(t, x(t)), \quad t=0,1, \ldots,
$$

where $A(t)=\left[\begin{array}{cc}0.5-e^{-t} & 0.4 \\ -0.4 & 0.5-e^{-t}\end{array}\right]$, and $F: Z^{+} \times R^{2} \rightarrow R^{2}$ is a given function satisfying the growth condition: there is a constant $\gamma>0$ such that

$$
\|F(t, h)\| \leq \gamma\|h\|, \quad \forall h \in R^{2} .
$$

If $A(t)=D(t)+V(t)$, where $D(t)=\operatorname{diag}\left(0.5-e^{-t}, 0.5-e^{-t}\right)$, and $V(t)=\left[\begin{array}{cc}0 & 0.4 \\ -0.4 & 0\end{array}\right]$, then we have

$$
\begin{aligned}
\rho_{0} & =\sup _{t=0,1, \ldots}\left(r_{S}(D(t))+\||V(t)|\|\right) \\
& =\sup _{t=0,1, \ldots}\left(0.5-e^{-t}+0.4\right)=0.9 .
\end{aligned}
$$


On the other hand, by choosing a suitable matrix norm, we obtain

$$
\|A(t)-A(s)\| \leq 0.5\left|e^{-t}-e^{-s}\right|, \quad \forall t \geq s \geq 0 .
$$

By the mean value theorem we have, for some $\tau \in[s, t]$ and $t \geq s$,

$$
\left|e^{-t}-e^{-s}\right|=e^{-\tau}|t-s|
$$

Hence, the function $q(t)=0.5 e^{-t}$ satisfies the conditions of Theorem 14; that is,

$$
\sum_{t=0}^{\infty}(q(t)+\gamma) \rho_{0}^{t}<1 .
$$

Consequently, by Theorem 14, the zero solution of system (68) is exponentially stable.

Remark 16. An efficient method to construct difference systems with slowly time-varying matrix functions is using a weaker condition than (20), namely, the uniform Lipschitz condition

$$
\|A(t+h)-A(t)\| \leq \beta(t)\|h\|^{\eta}, \quad \forall t \text { and some } \eta, 0<\eta \leq 1,
$$

with $\beta(t)$ small enough (see [16]).

\section{Conclusion}

The stability analysis of discrete-time-varying systems is harder than the stability analysis of time-invariant systems, because the stability and exponential stability of time-varying systems are not characterized by the spectrum of the transition matrices. Several approaches have been proposed in the literature to reduce its stability analysis to the analysis of related time-invariant systems $[1,2,7-9,11,18,22]$. The "freezing" technique has become well known among these techniques. In particular, it has been used to prove that exponential stability of a discrete-time-invariant system implies the exponential stability of the original (time-variant) system provided that the original linear system varies sufficiently slowly and a correct eigenvalue placement in the complex plane. The main novelty of our work is that we use estimates for the absolute values of entries of matrix-valued functions instead of bounds on its eigenvalues. Thus, we establish new stability results for time-varying systems with nonlinear perturbations which complement the existing literature concerning this subject. Natural directions for future research are the generalizations of our results to time-varying delay systems as well as to the stabilization of discrete control systems using new estimates to the norm of the powers of operators.

\section{Conflict of Interests}

The author declares that there is no conflict of interests regarding the publication of this paper.

\section{Acknowledgments}

The author is grateful to the anonymous referees for their careful review and encouraging comments. This research was supported by Fondo Nacional de Ciencia y Tecnologia (Fondecyt), Chile, under Grant no. 1130112.

\section{References}

[1] C. A. Desoer, "Slowly varying discrete system $x_{i+1}=A_{i} x_{i}$," Electronics Letters, vol. 6, no. 11, pp. 339-340, 1970.

[2] L. Jetto and V. Orsini, "Relaxed conditions for the exponential stability of a class of linear time-varying systems," IEEE Transactions on Automatic Control, vol. 54, no. 7, pp. 1580-1585, 2009.

[3] R. P. Agarwal, H. B. Thompson, and C. C. Tisdell, "Difference equations in Banach spaces. Advances in difference equations IV," Computers \& Mathematics with Applications, vol. 45, pp. 1437-1444, 2003.

[4] R. Memarbashi, "Sufficient conditions for the exponential stability of nonautonomous difference equations," Applied Mathematics Letters, vol. 21, no. 3, pp. 232-235, 2008.

[5] V. N. Phat and T. T. Kiet, "On the Lyapunov equation in Banach spaces and applications to control problems," International Journal of Mathematics and Mathematical Sciences, vol. 23, no. 3, pp. 155-166, 2002.

[6] V. Sundarapandian, "Exponential stabilizability and robustness analysis for discrete-time nonlinear systems," Applied Mathematics Letters, vol. 18, no. 7, pp. 757-764, 2005.

[7] F. Amato, G. Celentano, and F. Garofalo, "New sufficient conditions for the stability of slowly varying linear systems," IEEE Transactions on Automatic Control, vol. 38, no. 9, pp. 14091411, 1993.

[8] L. Jetto and V. Orsini, "Relaxed sufficient conditions for the stability of continuous and discrete-time linear time-varying systems," in Proceedings of the 46th IEEE Conference on Decision and Control, New Orleans, La, USA, December 2007.

[9] E. W. Kamen, P. P. Khargonekar, and A. Tannenbaum, "Control of slowly-varying linear systems," IEEE Transactions on Automatic Control, vol. 34, no. 12, pp. 1283-1285, 1989.

[10] A. Lasarow and D. Szegö, "Pairs of sequences of rational matrixvalued functions," International Journal of Mathematics and Mathematical Sciences, vol. 2006, Article ID 23723, 37 pages, 2006.

[11] M. I. Gil and R. Medina, "The freezing method for linear difference equations," Journal of Difference Equations and Applications, vol. 8, no. 5, pp. 485-494, 2001.

[12] R. Medina and M. I. Gil, "Accurate solution estimates for nonlinear nonautonomous vector difference equations," Abstract and Applied Analysis, vol. 2004, no. 7, pp. 603-611, 2004.

[13] R. Medina, "Exponential stabilization of nonlinear discretetime systems," Journal of Difference Equations and Applications, vol. 17, no. 5, pp. 697-708, 2011.

[14] R. Medina, "Stabilizability for nonlinear systems of difference equations," International Journal of Robust and Nonlinear Control, vol. 20, no. 10, pp. 1156-1165, 2010.

[15] R. Medina, "Global stabilization of non-linear discrete-time systems by linear feedback," Journal of Mathematical Control and Information, vol. 25, no. 3, pp. 341-351, 2008.

[16] V. Solo, "On the stability of slowly time-varying linear systems," Mathematics of Control, Signals, and Systems, vol. 7, no. 4, pp. 331-350, 1994. 
[17] E. D. Sontag, Mathematical Control Theory. Deterministic Finite Dimensional Systems, Springer, New York, NY, USA, 1990.

[18] F. Wirth and D. Hinrichsen, "On stability radii of infinitedimensional time-varying discrete-time systems," Journal of Mathematical Control and Information, vol. 11, no. 3, pp. 253276, 1994.

[19] S. Wu, C. Li, X. Liao, and S. Duan, "Exponential stability of impulsive discrete systems with time delay and applications in stochastic neural networks: a Razumikhin approach," Neurocomputing, vol. 82, pp. 29-36, 2011.

[20] M. I. Gil', "Estimates for absolute values of matrix functions," Electronic Journal of Linear Algebra, vol. 16, pp. 444-450, 2007.

[21] L. Verde-Star, "Functions of matrices," Linear Algebra and Its Applications, vol. 406, no. 1-3, pp. 285-300, 2005.

[22] J. Diblík and D. Y. Khusainov, "Representation of solutions of discrete delayed system $x(k+1)=A x(k)+B x(k-m)+f(k)$ with commutative matrices," Journal of Mathematical Analysis and Applications, vol. 318, no. 1, pp. 63-76, 2006. 


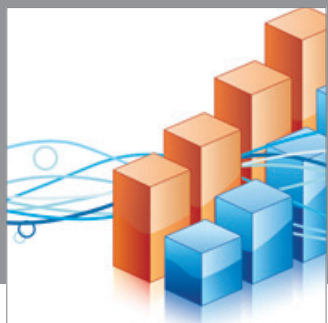

Advances in

Operations Research

mansans

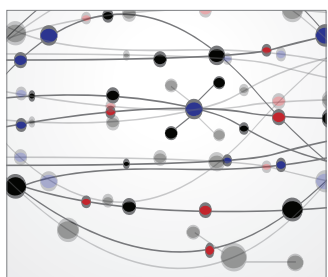

The Scientific World Journal
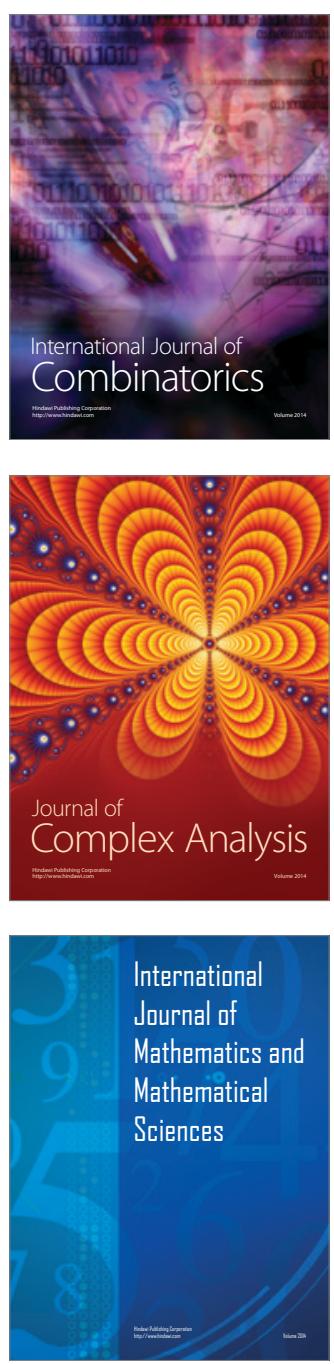
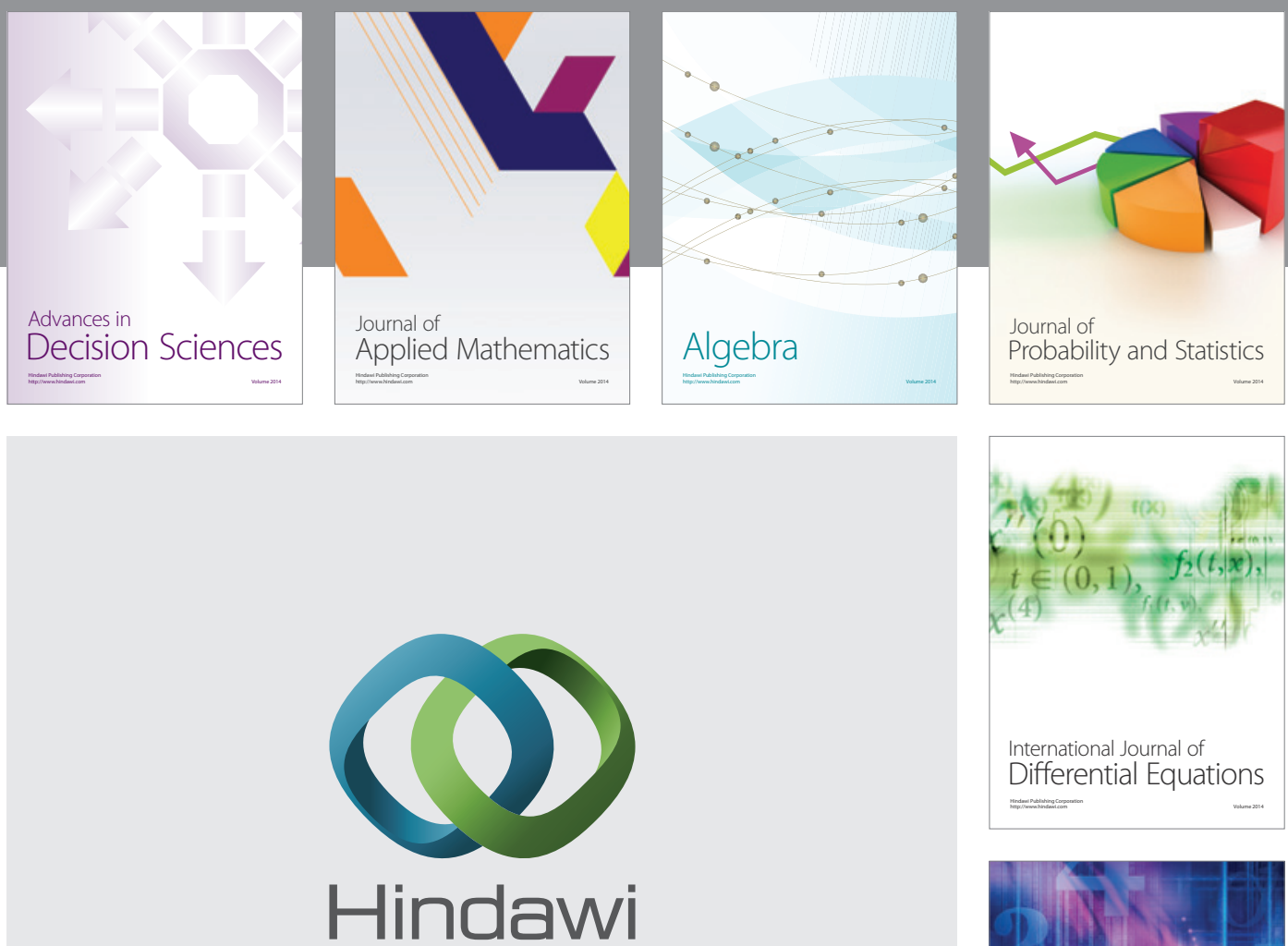

Submit your manuscripts at http://www.hindawi.com
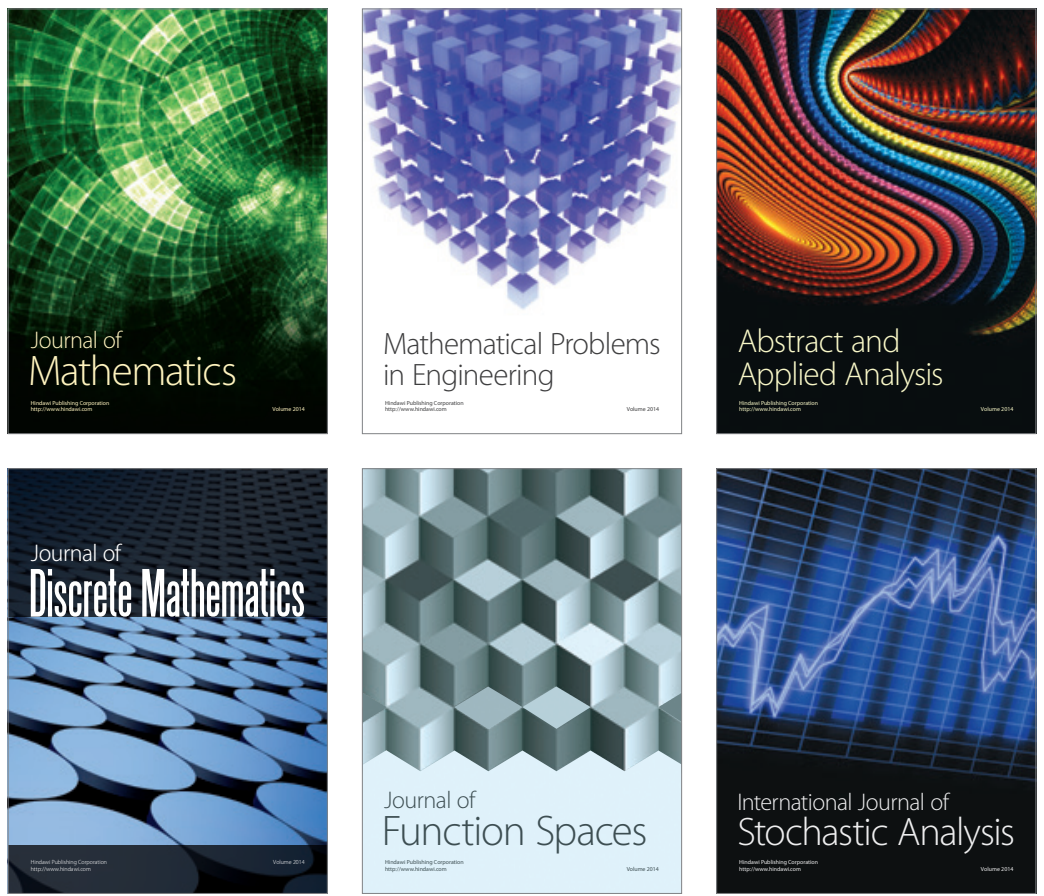

Journal of

Function Spaces

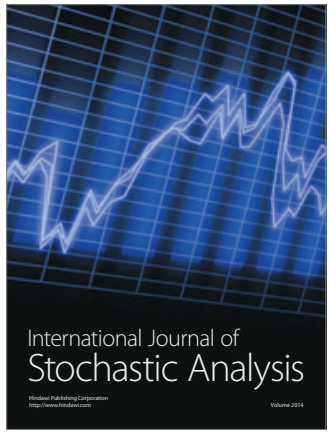

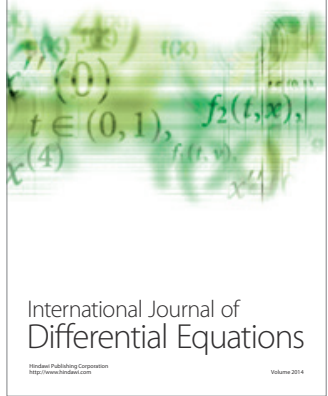
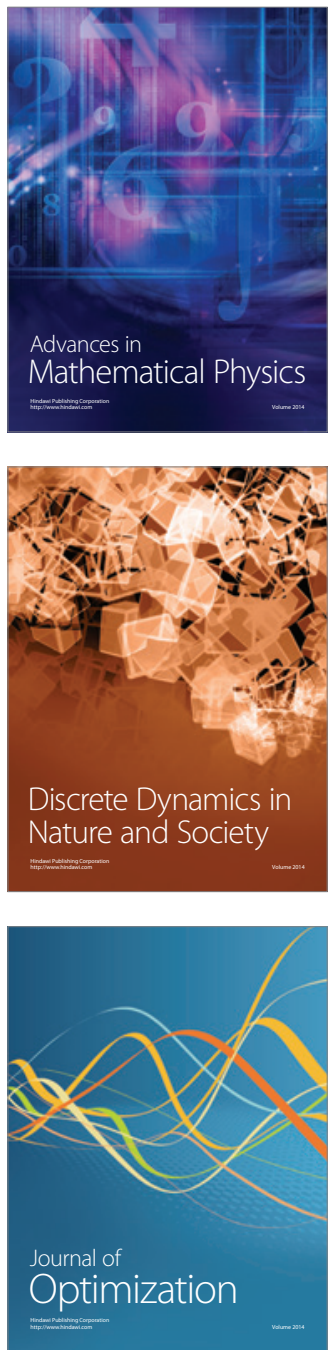